\title{
CcMP-II Metalloproteinase from Cerastes Cerastes Snake Venom Acts both on Blood Vessel ECM and Platelets
}

Type of article: Conference abstract

Amel Bennacer, Hinda Boukhalfa-Abib, Fatima Laraba-Djebari

USTHB, Faculty of Biological Sciences, Laboratory of Cellular and Molecular Biology, BP 32, El-Alia

Bab Ezzouar, 16111, Algiers, Algeria

Corresponding Author: amel4903@g mail.com

\begin{abstract}
:
Background: Snake venoms contain various metalloproteinases that are highly toxic, inducing in severe bleeding by interfering with the blood coagulation and degrading the basement membrane or extracellular matrix (ECM) components. It has been suggested that hemorrhagic metalloproteinases interact in a specific way with platelet surface proteins resulting in an alteration of platelet function. Hemorrhage and tissue necrosis are common manifestations of viper envenomations in humans, largely due to the actions of prominent metalloproteinases. CcMP-II a weak hemorrhagic metalloproteinase purified from Cerastes cerastes venom belongs to the class P-II snake venom metalloproteinase.

Methods: In this study, we characterized the biological effects of CcMP-II, including the ability of the enzyme to hydrolyze extracellular matrix components and plasma proteins, as well as its histopathological effects induced in capillaries. Then a study of the anti-aggregating activity was carried out and controlled for 10 min using our Chrono-log aggregometer (ServiBIO) (USTHB, Faculty of Biological Sciences, BP 32, El-Alia Bab Ezzouar, 16111, Algiers, Algeria in 2019). The histopathological changes induced by CcMP-II were also analyzed and the $1 \mu \mathrm{m}$ semi-thin sections are then stained with Toluidine Blue and the observation of these sections was realized using a photomicroscope. Toluidine Blue, magnification: 100x.

Results: Obtained results showed that CcMP-II metalloproteinase hydrolyzed selectively the A $\alpha$ chain of fibrinogen. This enzyme hydrolyzed also laminin and type IV collagen in time- and dosedependent manner. CcMP-II inhibits collagen-induced platelet aggregation of human PRP with an $\mathrm{IC}_{50}$ value of $0.11 \mathrm{nM}$. Pathological changes induced by CcMP-II after intramuscular injection in mice gastrocnemius were also studied. Histological study showed that the main morphological alterations in capillary vessels are characterized by a separation of endothelial cells from the surrounding basement membrane and reduction in the width of these cells, together with loss of basement membrane in some areas, through which erythrocytes and plasma are extravasated.

Conclusion: In conclusion, CcMP-II, as a member of the P-II class of SVMPs, presents a selective specificity toward fibrinogen, laminin and type IV collagen. It was an $\alpha$ - fibrinogenase metalloproteinase, which inhibits collagen-stimulated platelet aggregation. This study will contribute to understanding better the functional mechanisms of the metalloproteinases that may be useful in the development of therapeutic agents related to thrombotic disorders.
\end{abstract}

Keywords: Snake Venom, CcMP-II, Metalloproteinase, Platelets, Capillary.

\section{Conflict of interest statement}

This article is a conference abstract presented at the International Congress on Health Sciences and Medical Technologies, Tlemcen Algeria 5-7 December 2019, ICHSMT'19.

\section{Authors' biography}

No Biography. 
Medical Technologies Journal, Volume: 4, Issue: 1, January-March 2020, Pages: 516-517. Doi :

https://doi.org/10.26415/2572-004X-vol4iss1p516-517

\section{References}

No references 